INTERNATIONAL DESIGN CONFERENCE - DESIGN 2018

https://doi.org/10.21278/idc.2018.0122

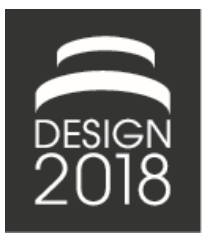

\title{
SUPPORTING ENGINEERS IN LIGHTWEIGHT DESIGN: THE ENERGY DISTRIBUTION ANALYSIS (EDA)
}

\author{
F. Laufer, D. Roth and H. Binz
}

\begin{abstract}
Lightweight design methods help the engineer to design lighter products. However, none of the existing methods support lightweight design with regard to the state of motion of mass and the mass distribution. This paper presents an analytic method to fill this gap. The method uses kinetic and potential energies to determine an energy level factor. This factor enables the engineer to derive an optimization potential and order of all different product assemblies. Finally, a case study is performed on a processing machine to illustrate the effectiveness of the developed method.
\end{abstract}

Keywords: lightweight design, design methods, design for $x(D f X)$, conceptual design

\section{Introduction}

Lightweight design is a key factor in today's engineering practice for the design of competitive products which fulfil the customer's needs on the one hand and satisfy economic and environmental requirements on the other. Lightweight strategies are powerful tools when it comes to supporting engineers in designing light products. These strategies are spread over the entire product-development process, addressing the different stages from task clarification through to embodiment design (Henning and Moeller, 2011). Most of the product characteristics are determined during the conceptual design phase and thus have great potential to reduce the weight of a product (Ponn and Lindemann, 2011). The decision to power a car with either an electric motor or a combustion engine, for instance, forms part of the conceptual design phase and significantly affects the weight of the car. One lightweight strategy in this phase is conditional lightweight design, which takes into account different requirements for the product such as cost, $\mathrm{CO} 2$ balance or crash regulations (Henning and Moeller, 2011). A method within this strategy is target weighing (Ponn and Lindemann, 201; Posner et al., 2012; Posner et al., 2013; Albers et al., 2013; Albers et al., 2017), which focuses on the target mass that a product must attain, or alternatively the mass that a product must lose. This paper aims to increase the methodological support for lightweight design in the conceptual design phase, and presents a method for analyzing the potential of lightweight design with regard to the state of motion of mass and a product's distribution of mass.

\section{Problems and goals}

When reducing the mass of a product, it is prudent to set a final target mass. To reach this goal, different lightweight strategies and methods can be applied to a certain product, whereby the method is a rulebased procedure for realizing the aim of the strategy. However, existing methods neither point out a component to optimize nor identify a suitable component to reduce weight with respect to the state of motion of mass and mass distribution. Attaining the overall target mass by applying different lightweight 
methods randomly to different components may lead to unintentional mass-related behavior. If, for instance, the center of gravity moves upward due to a randomized mass reduction of a car, the driving characteristics might be adversely affected.

With a view to the conceptual design phase in the product-development process, there is a gap in lightweight design methodology regarding the mass, the state of motion of mass and the distribution of mass. Posner et al. (2012) contended that a holistic lightweight design methodology has to take these things into account. Posner et al. (2013) elaborated by presenting the Function Mass Analysis, in which the value analysis is operationalized for lightweight design by transferring the application in terms of mass instead of costs. The result is a method which enables the actual mass and the target mass of each component to be compared with respect to the customer's requirements, thus identifying the lightweight optimization potential. However, a methodological approach for mass reduction with respect to the state of motion of mass and mass distribution is still lacking. This results in the following hypothesis: "It is possible to identify and quantify the mass-reduction potential of a product with respect to the state of motion of mass and the mass distribution with a method." The objective of this paper is thus to present such a method, thereby filling the corresponding gap in lightweight design methodology for the conceptual phase.

\section{State of the art}

There is a large amount of literature addressing lightweight design strategies, e.g. material lightweight design, in addition to lightweight design methods, e.g. shape optimization using topology-based methods (refer to Henning and Moeller, 2011 and Klein, 2013 for an exhaustive review). To exploit the great potential in terms of the weight of a product during the conceptual design phase, Feyerabend (1990) transferred the Value Analysis (VDI, 2010) from its application in terms of costs to application in terms of mass in order to reduce the weight of a robot. Furthermore, he used the mass moment of inertia to analyze and derive mass-reduction potential. Posner et al. (2013) operationalized the procedure by presenting the Function Mass Analysis and making it applicable to any kind of product. In addition, Albers et al. (2017) introduced an extended approach to take CO2 emissions and costs into account for a holistic lightweight design. Nonetheless, a method which holistically considers the state of motion of mass and mass distribution is still lacking.

Another way to address lightweight design in terms of its mass and distribution during the conceptual design phase is to study car and airplane design.

Different authors propose lightweight zones in car design. These zones take different criteria such as costs and traction into account, resulting in classification by factor of the lightweight potential throughout the car (Haldenwanger, 1997; Friedrich, 2013).

As a sub-discipline of car design, race car design has even higher standards concerning weight limits and mass distribution. Here, the so-called "packaging" describes the arrangement of the assemblies within a car's structure. Every race car design problem results in a trade-off for arranging the different parts, e.g. prioritizing a complex aerodynamic structure over car weight to a certain extent. One primary aim for mass distribution is to make the overall center of gravity as low as possible (Trzesniowski, 2014).

In airplane design, the approximate weight of every section is determined from previous models. After assigning the weight values to the various design departments as a starting point for further evaluation, the design weight is not significantly altered at a later stage. The design layout should then be carefully optimized; hence, a high accuracy of the initial weight prediction is a prerequisite. The primary aim here is to design the center of gravity in such a way that good stability and control properties are attained (Torenbeek, 1976).

When summarizing the design guidelines for cars and airplanes in terms of mass distribution, the design process presents itself as a highly iterative procedure. This procedure cannot be generalized due to the high number of different criteria considered.

\section{The Energy Distribution Analysis}

In this section, the new "Energy Distribution Analysis (EDA)" method is presented. Firstly, the requirements for an engineering-design method are discussed. Secondly, the precise aim of the EDA is 
elaborated. Thirdly, the rule-based procedure is explained and visualized with a flow chart. In the following section, the method is then applied to a technical system (a 2-axis processing machine) to illustrate the step-by-step procedure in a case study.

\subsection{Overarching requirements for a method}

Overarching requirements for engineering-design methods in general were published by Keller and Binz (2009). The method presented here claims to fulfil the criteria "practical relevance \& competitiveness", "scientific soundness", "problem specificity" as well as "structure \& compatibility". By applying the method to different products in different industrial environments, the requirements "revisability", "comprehensibility", "usefulness" and "flexibility" need to be evaluated in a subsequent step.

\subsection{Specifying the aim of the method}

The overarching objective of the method presented in this paper is to make a contribution to faster and more efficient development of lightweight products. This must be achieved by determining different mass-reduction potential values for the different components in a product, thus deriving an order of mass optimization. The method will not include suggestions for alternative placement of components, however. To support the engineer in innovative lightweight design during the early phases, the method must take different points into account. According to Posner et al. (2012), there are five significant, specific requirements:

- Consideration of function

- Consideration of mass

- Consideration of state of motion of mass (mass moment of inertia)

- Consideration of mass distribution

- Consideration of mass-dependent working principle

By developing the Function Mass Analysis, Posner et al. (2013) took the function and mass into consideration. Considering this and the state of the art presented in Section 3, there is still no operationalized support in the form of a design method for the state of motion of mass (mass moment of inertia), mass distribution and mass-dependent working principle. The following subsections examine these three requirements in greater detail in accordance with Posner et al. (2012).

\subsubsection{Consideration of state of motion of mass}

Posner et al. (2012) note that a holistic method for function and mass needs to respect the mass moment of inertia. However, the mass moment of inertia only takes rotational motions into account. A holistic method must respect both the rotation and translation of a body. The consideration of the state of motion of mass replaces this requirement capturing all states of motion of mass. That aside, a body might not be subject to motion, but might rather be in a static state. These motions of different product parts have a great influence on the energy consumption of the product. A method should thus be able to make a statement concerning the optimization potential of a component by taking the state of motion of that particular component into account. The differentiation between rotation and translation is the first thing to consider here.

Secondly, the various kinds of motion for a body, such as motion with constant velocity $v[\mathrm{~m} / \mathrm{s}]$ or accelerated motion $a\left[\mathrm{~m} / \mathrm{s}^{2}\right]$, are measured in different units. A method must be capable of dealing with these different motions and finding a suitable physical quantity to cover all kinds of differently moving components on one level.

Thirdly, it is necessary to keep the overall target mass in mind. When, for instance, the overall mass of a tooling machine is reduced by $30 \%$, the lightweight optimization potential of the spindle is potentially higher than that of the slower-moving machine bench due to the former's relatively high rotation speed. However, this may prevent the overall target mass from being achieved because the machine bench represents a substantial proportion of the total machine mass. The mass $m$ of each component therefore has to be considered as well. 
A physical quantity which is able to combine all these considerations is kinetic energy. For translatory motions, the kinetic energy is (Hering et al., 2007 for all equations):

$$
E_{k i n}^{\text {trans }}=\frac{1}{2} m v^{2}
$$

Here, $m$ is the mass and $v$ the velocity of the component. Furthermore, the kinetic energy of a rotatory motion is:

$$
E_{\text {kin }}^{r o t}=\frac{1}{2} J_{P} \omega^{2}
$$

Here, $J_{P}$ is the mass moment of inertia and $\omega$ is the angular velocity of the component. $J_{P}$ is represented by the integral:

$$
J_{P}=\int r^{2} d m
$$

Here, $r$ is the radius to the rotation axis $P$ with continuous mass distribution within the rotating body. The total kinetic energy level of a component can be calculated by the sum of its translation and rotation energy:

$$
E_{\text {kin }}^{\text {total }}=E_{\text {kin }}^{\text {trans }}+E_{\text {kin }}^{\text {rot }}
$$

Consequently, a method can use the kinetic energy level $E_{\text {kin }}$ of a certain component to derive optimization potential.

\subsubsection{Consideration of mass distribution}

A lightweight design method during the early phases of the product development process must also take the mass distribution into account. This is necessary due to the unintentional mass-related behavior that a randomized mass reduction of different components can have.

To consider the distribution of different components within the product, the center of gravity (c.g.) of each component represents the point around which the resultant torque caused by gravitational forces vanishes (Hering et al., 2007). The distance between the c.g. of each component to the total c.g. of the product enables the relative position of all components to be quantified. This consideration results in the first factor, $h$, which concerns the c.g. distances. A method should thus be able to make a statement concerning the different optimization potential of each component based on $h$, e.g. in terms of a shift of the total c.g. of a car downward. In this case, the car roof would have a higher potential than the seats.

Secondly, as with the consideration of state of motion of mass, a method addressing the distribution of mass must consider the overall target mass. Using the example of the car, it must be considered that all seats can gather more mass than the car roof, while the roof may have a higher optimization potential than the seats. Here, too, $m$ represents the mass of each individual component of a product.

A third point to consider is the c.g. of the components in relation to each coordinate axis $(\mathrm{x}, \mathrm{y}, \mathrm{z})$. A literature analysis revealed that there are two different types of aim regarding the location of the overall c.g. of a product. On the one hand, there is a goal of shifting the c.g. toward an extremum. The axis considered in this case is frequently the vertical axis (z) along the gravitational field (Torenbeek, 1976; Trzesniowski, 2014). The idea behind this is to improve the product characteristics in terms of an acting force which tends to unbalance the product, e.g. the centrifugal force on a car driving into a bend. In this example, a low c.g. in terms of $z$ allows higher cornering speeds (Trzesniowski, 2014). On the other hand, considering the c.g. with respect to the horizontal axes (x, y) orthogonal to the gravitational field, the goal often manifests itself as a balance of the product. Therefore, the c.g. must be allocated inside a particular zone, e.g. for a well-balanced axle-load distribution on a car in a zone between both axles. This improves the traction, depending on the location of the engine and the drive axle (Trzesniowski, 2014). Figure 1 illustrates the two different kinds of destination of the c.g. 


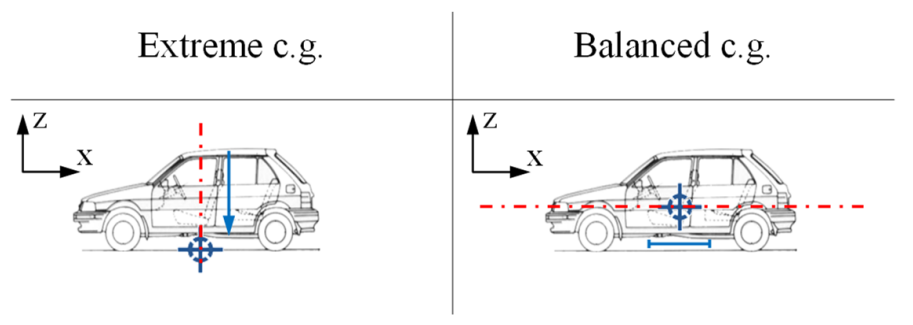

\section{Figure 1. Different destinations of the center of gravity (blue cross) on a car depending on the considered axis (red dotted line)}

In the case of a balanced c.g., an optimization has to take the mass of two different components into account in order to maintain that balance. This necessitates special design considerations and will be the objective of another paper. As a result, this paper solely focuses on the c.g. location with respect to the vertical axis (z).

The physical quantity $E_{\text {pot }}$ considers the distance, $h$, the mass, $m$, as well as the distribution of mass with respect to the vertical axis (with gravitational constant $g$ ) (Hering et al., 2007):

$$
E_{p o t}=m g h
$$

Consequently, a method can use the potential energy level $E_{\text {pot }}$ of a certain component to derive its optimization potential.

\subsubsection{Consideration of mass-dependent working principle}

The appropriateness of the mass reduction of single components within a certain product may differ among those components. Therefore, the method should be able to evaluate the different components based on their determined energy level with regard to their mass-dependent working principle.

The reduction of the mass moment of inertia of a turbine stage by reducing the masses of the blades, for instance, is reasonable to save energy. Conversely, it is also reasonable to keep the overall mass moment of inertia of a hand-held drill high so as to reduce the resulting torque forces on the wrist of the user. In order to achieve this objective, the relatively heavy battery of a hand-held drill should be as far away as possible from the rotating axis of the drill chuck, thus it is usually placed at the very end of the handle. Another example is shown in Figure 2.

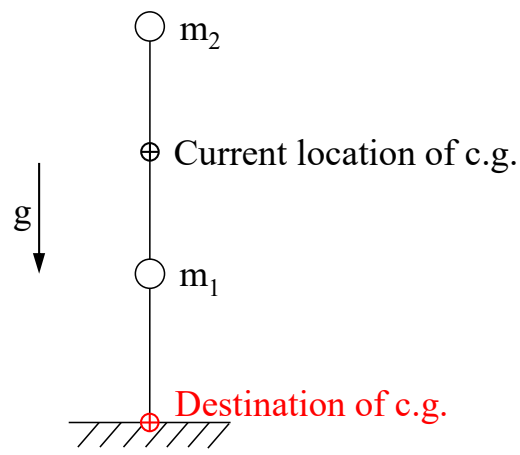

Figure 2. Shifting the location of the c.g. downward along the vertical axis

When reducing the total mass of a two-mass system with the aim of shifting the total c.g. downward along the vertical axis, the two masses have to be approached differently. By reducing the upper mass $\left(\mathrm{m}_{2}\right)$, the total c.g. shifts downward in the desired direction. By reducing the lower mass $\left(\mathrm{m}_{1}\right)$ instead, the total c.g. moves upward, which is contrary to the aim.

One means of approaching these different mass-dependent working principles is to assign the determined energy level of each component a positive or a negative sign. This helps to determine whether it is more reasonable to reduce the mass of that particular component, i.e. $+E$, or less reasonable to reduce the mass of that particular component, i.e. - $E$. However, this nomenclature does not mean that 
a component with a negative energy level $(-E)$ should not be reduced at all. The overall target mass in mind, it indicates that this particular mass should be reduced with a lower priority than other components with a positive energy level $(+E)$. Referring to the example in Figure 2, mass 2 has a positive potential energy level $\left(+E_{\mathrm{pot}}\right)$, while mass 1 has a negative energy level $\left(-E_{\mathrm{pot}}\right)$. In order to measure the potential energy level correctly, however, it is necessary to take the current total c.g. of the system as a reference level.

\subsubsection{Making a statement on the different optimization potential using the component energies}

All of the previous considerations lead to a total energy level for each individual component, which is calculated from its potential energy $E_{\mathrm{pot}}$ (every component) and its kinetic energy $E_{\text {kin }}$ (only moving components). Figure 3 illustrates the different energy levels in a technical system with three components. The individual component energy level then appears to be positive or negative overall.

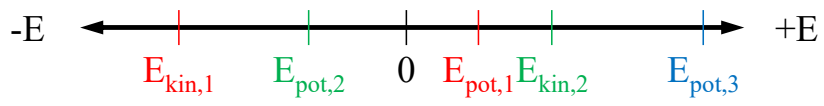

Figure 3. Different energies of three different components

To compare the different energy levels of $n$ components with each other, the relative amount of the component's energy level to the total energy of the technical system is described by the so called energy level factor $\eta_{i}$ :

$$
\eta_{i}=\frac{ \pm E_{k i n, i}^{\text {total }} \pm E_{p o t, i}}{\sum_{i}^{n}\left(\left|E_{k i n, i}^{\text {total }}\right|+\left|E_{p o t, i}\right|\right)}
$$

Here, the numerator represents the individual component's energy level, which may be either positive or negative, while the denominator represents the total energy level of the whole technical system. This level must be positive to capture the absolute quantity of energy in the system. The energy-level factor therefore appears to be within the range of $-1 \leq \eta_{i} \leq 1$. As with the energy level of a component, the energy-level factor of a component indicates the preferred degree of optimization. Here, as shown in Figure 4 , a $\eta_{i}$ close to 1 displays a major preference, whereas a $\eta_{i}$ close to -1 displays a minor preference for optimizing the concerned component with regard to lightweight.

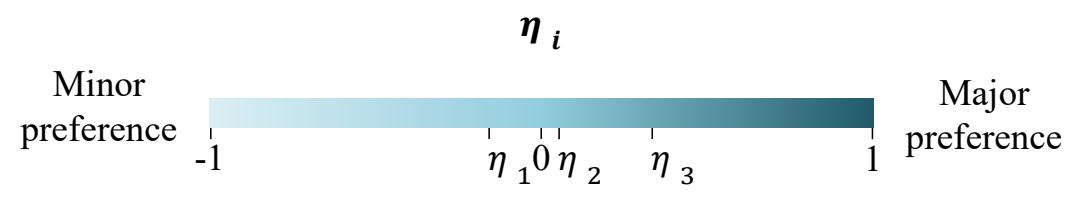

Figure 4. Optimization preference depending on the energy-level factor $\eta_{i}$ of the three different components from Figure 3

Besides identifying the mass-reduction potential, the hypothesis introduced in Section 2 also requires a quantification of these potential values. Based on $\eta_{i}$, the actual mass loss to be reached for every chosen component therefore has to be determined. To consider the energy-level factor within the mass-loss calculation, the actual mass that a component has to lose must be addressed. The overall mass-loss factor $\alpha$, which indicates the overall mass loss of the product, can be broken down into a component level and measures between $0 \%$ and $100 \%$. The factor itself then appears inside the range of $0 \leq \alpha \leq 1$. Based on $\alpha$, the mass loss at a component level is:

$$
C_{i, \text { new }}=C_{i, \text { old }}(1-\alpha)=C_{i, \text { old }}-C_{i, \text { old }} \alpha
$$


Here, $C_{i}$ is the mass of a component. Establishing $\eta_{i}$ on the right-hand side of Equation 7 yields the following:

$$
C_{i, \text { new }}{ }^{\prime}=C_{i, \text { old }}-C_{i, \text { old }} \alpha \eta_{i}
$$

Here, $\eta_{i}$ now affects the mass loss of every component. Looking at the second term on the right-hand side of Equation 8 , negative $\eta_{i}$ values with a minor optimization preference now increase $C_{i, \text { new }}$ ' Conversely, positive $\eta_{i}$ values with a major optimization preference decrease $C_{i, n e w}$ ' . This means that a component with a minor preference for mass reduction becomes heavier in relation to a component with a major preference. The placement of $\eta_{i}$ in Equation 8 is thus consistent with the aim of the approach, although the integration of $\eta_{i}$ into the mass-loss calculation influences the total amount of reduced mass, making $C_{i, n e w}$ to an artificial component mass. As a consequence, the relative amount of Equation 8 has to be formed to respect the overarching target mass. Doing so yields the following:

$$
\vartheta_{i}=\frac{C_{i, \text { old }}-C_{i, \text { old }} \alpha \eta_{i}}{\sum_{i}^{n}\left(C_{i, \text { old }}-C_{i, \text { old }} \alpha \eta_{i}\right)}
$$

Subsequently, the mass-fraction factor, $\vartheta_{i}$, enables the targeted mass loss for each considered component to be determined. The factor is within the range of $0 \leq \vartheta_{i} \leq 1$, whereby all $\vartheta_{i}$ values add up to 1 due to the normalization in Equation 9. Through multiplication by the new total mass of the system, $S_{\text {new }}=S_{\text {old }}(1-\alpha)$, the new mass of each component is as follows:

$$
C_{i, \text { new }} "=S_{\text {new }} \vartheta_{i}
$$

Finally, $C_{i, n e w}$ represents the last result of a step-by-step procedure for determining a weighted massloss calculation with respect to the state of motion of mass, the mass distribution and the mass-dependent working principle of each component in a technical system.

\subsection{Procedure of the Energy Distribution Analysis (EDA)}

This section provides an overview of the step-by-step procedure of the EDA. In accordance with Posner et al. $(2012,2013)$ the EDA is inspired by the Function Mass Analysis with regard to its execution. As a method for lightweight design in early phases, the procedure is assigned to the product-design process within the conceptual phase.

To be able to determine the presented energy levels, the mass, motion sequences, centers of gravity and the distribution of mass of all components are required. Starting with this set of information for an existing product, e.g. a competitive product or a preceding product, the EDA is split into four steps. Firstly, the existing product must be analyzed in terms of its requirements from the customer's perspective. Unintentional mass-related behavior with respect to the mass-dependent working principle is also a point of interest. It must therefore be ascertained which parts of the product are working well with regard to mass and which are not.

Secondly, the boundary conditions for the lightweight optimizations must be defined. This is where the ideal location of the overall center of gravity is with respect to the vertical axis. The overall target mass must also be defined. A decision has to be made concerning which level of granularity the product must be examined at. This is possible both at an assembly level and a component level, depending on the acceptable accuracy and effort. 
Thirdly, the energy level of all chosen components/assemblies must be determined with the procedure presented in Section 4.2.

In the fourth and final step, the optimization potential and, consequently, the order of optimization priority must be derived based on the different mass-fraction factors. After this, the EDA is completed. The step-by-step procedure is illustrated in Figure 5.

According to Posner et al. (2013), the next step is to apply suitable lightweight approaches to the components in the derived order. As a consequence, an optimized component layout leads to several conceptual design solutions.

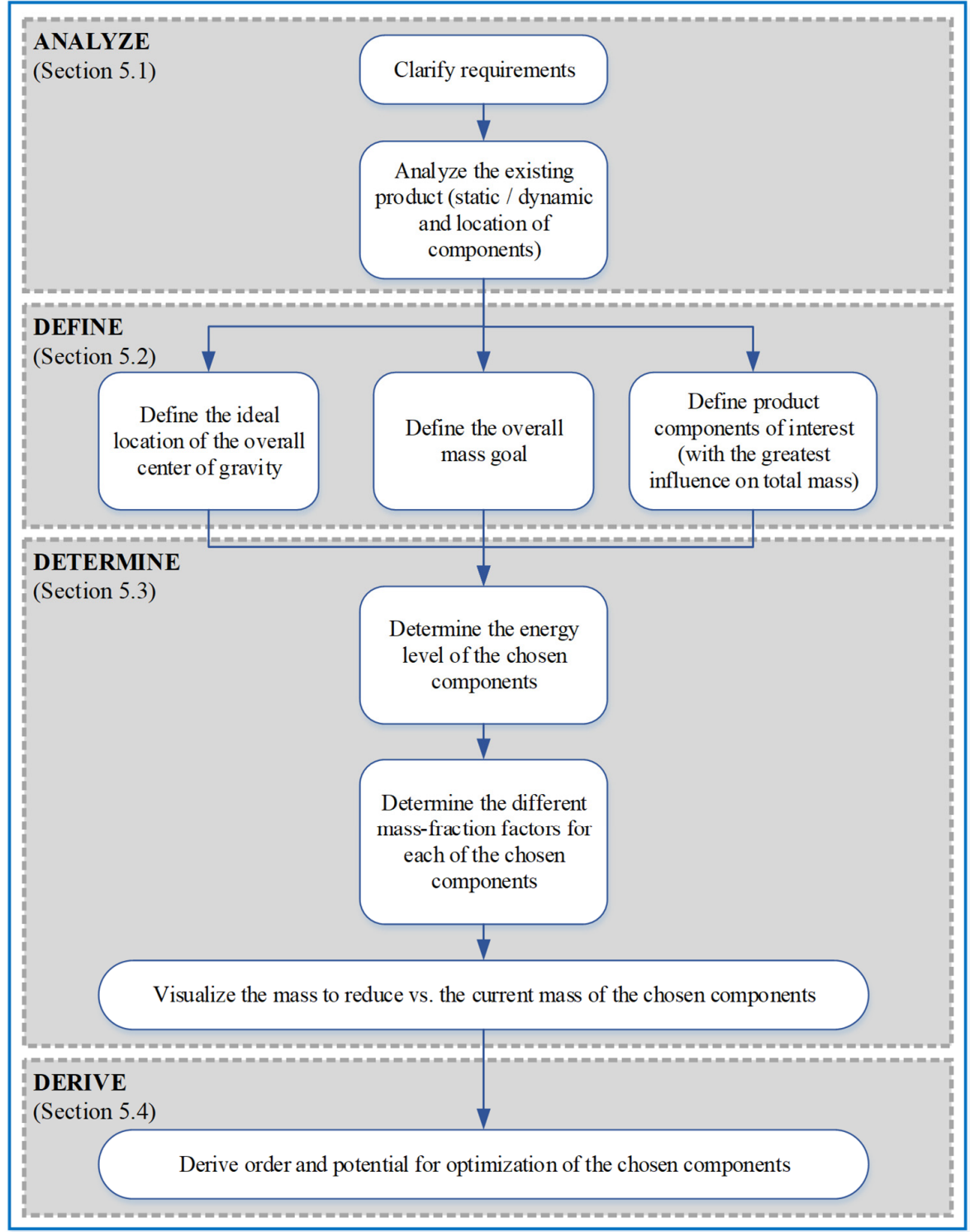

Figure 5. Flow chart showing the step-by-step procedure of the EDA

\section{Application and discussion}

In this section, the Energy Distribution Analysis is applied to the 2-axis processing machine shown in Figure 6. The system comprises a static frame that stands fixed on the ground. On top of the frame sits the motion unit, which is able to perform a 2-axis motion. The vertical cart, driven by a geared electric motor in combination with a belt, facilitates the vertical motion of the operating fork. The horizontal 
cart does the same for the horizontal motion and is powered in the same way. The processing machine operates inside the work space (limited by the frame dimensions) and carries stacks of cardboard boxes from one end to the other.

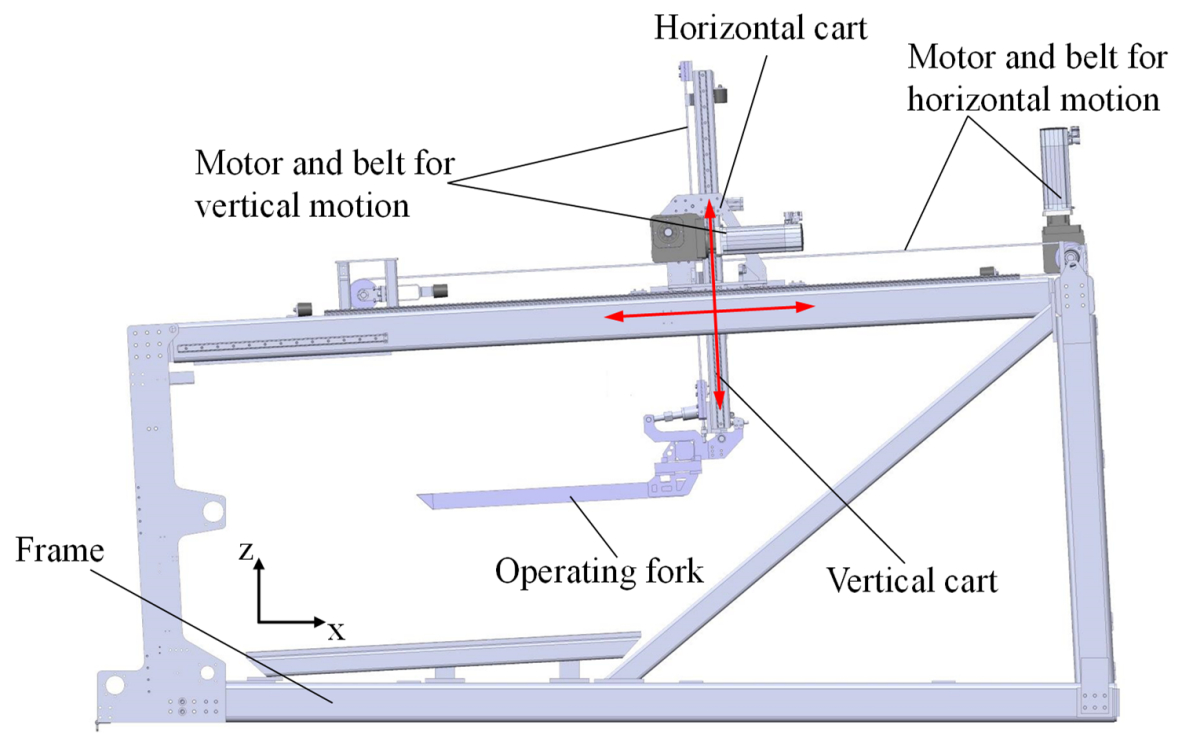

Figure 6. Side view of 2-axis processing machine with motion directions (red arrows)

\subsection{Analyzing the product}

When analyzing the product, several points appear to be critical with regard to mass. Firstly, the motion unit comprising the vertical and horizontal cart in addition to the operating fork causes the machine to oscillate. This is partly due to the fact that the accelerated mass of the motion unit exerts a certain force onto the frame. The location of the motion unit on top of the processing machine also leads to a long lever arm in terms of the machine's total center of gravity, thus causing high amplitudes of oscillation which reduce the service life of the product. To address these problems, the frame has been reinforced by several struts and more rigid connection points for the various frame components. This incurs higher costs.

The requirements derived from this analysis are to realize a lower total c.g. in terms of the vertical axis, to reduce the oscillation amplitude and to reduce the overall mass of the system to save costs.

\subsection{Defining the boundary conditions}

The boundary conditions are set according to the three points listed in Figure 5. The ideal location of the center of gravity is set at the very bottom of the tooling machine, thus helping to reduce oscillation and making the system more stable. The overall target mass is set at $70 \%$ of the mass of the existing product.

Finally, the decision must be made as to which components are to be investigated. The method enables the granularity of the considered system parts to be scaled. It is possible to examine the product in depth at an individual component level, yet also to examine it from an assembly perspective. In this case, the 2-axis processing machine will be examined on an assembly level, thus looking at the frame, the two carts and the operating fork. This is appropriate for an initial examination of the machine due to the depth of information derived from the analysis. In a subsequent step, it is possible to investigate the machine at a component level for more information.

\subsection{Determining energy-level factors and mass-fraction factors}

To determine the energy-level factor, $\eta_{i}$, Equations 1-6 are used as described above. The same is performed with Equations 7-9 for the mass-fraction factor, $\vartheta_{i}$. For reasons of confidentiality, the 
authors are not permitted to publish any concrete geometric data or velocity profiles for the 2-axis processing machine from Figure 6 . However, the total system mass is $2884 \mathrm{~kg}$. The frame is by far the heaviest assembly of the system, representing $86 \%$ of the total mass. Due to the fact that it is the only assembly not subject to motion and that the c.g. is below the total c.g. of the system (i.e. $-E_{\mathrm{pot}}$ ), the massreduction preference is negative (i.e. $-\eta_{i}$ ). Any other assembly has a positive $\eta_{i}$, either as a result of a high potential or high kinetic energy level. The results are shown in Table 1, whereby the overall aim is considered to be a $30 \%$ reduction in mass for the entire product, resulting in a new total product mass of $2018.8 \mathrm{~kg}$. In the last column $\beta$ represents the new weighted percentage mass loss of each individual component.

Table 1. Energies, energy-level factors and mass-fraction factors of all assemblies

\begin{tabular}{lrrrrrrrr}
\hline Assembly & $\mathrm{m}[\mathrm{kg}]$ & $\alpha[\%]$ & $E_{\text {kin }}^{\text {total }}[\mathrm{J}]$ & $E_{\text {pot }}[\mathrm{J}]$ & $\sum E[\mathrm{~J}]$ & $\eta_{i}$ & $\vartheta_{i}[\%]$ & $\beta[\%]$ \\
\hline Frame & 2480 & 30 & - & -4226.2 & -4226.2 & -0.290 & 88.09 & 28 \\
Horizontal cart & 229 & 30 & 3297.6 & 2339.0 & 5636.6 & 0.387 & 6.61 & 42 \\
Vertical cart & 145 & 30 & 2088.0 & 2069.4 & 4157.4 & 0.285 & 4.33 & 40 \\
Operating fork & 30 & 30 & 432.0 & 115.1 & 547.1 & 0.038 & 0.97 & 35 \\
\hline
\end{tabular}

Offsetting the mass-fraction factors, $\vartheta_{i}$, in the last column in Table 1 with the new total product mass as specified in Equation 10 results in the new assembly masses shown in Figure 7.

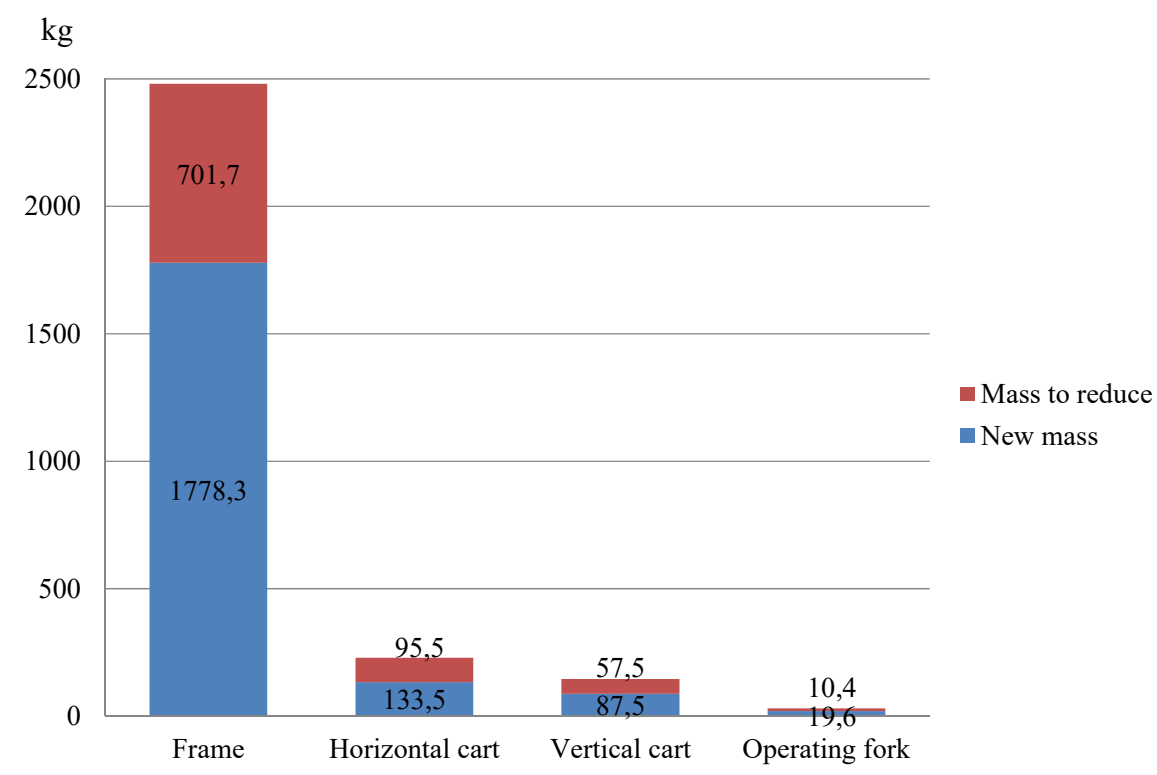

Figure 7. New masses of the assemblies (blue bar) with mass to reduce (red bar on top)

\subsection{Deriving order and potential for optimization}

The different energy levels result in the different quantities of mass that the assemblies have to lose, as shown in Figure 7. Another way to display the final result with the weighted percentage mass loss $\beta$ is illustrated in Figure 8. 


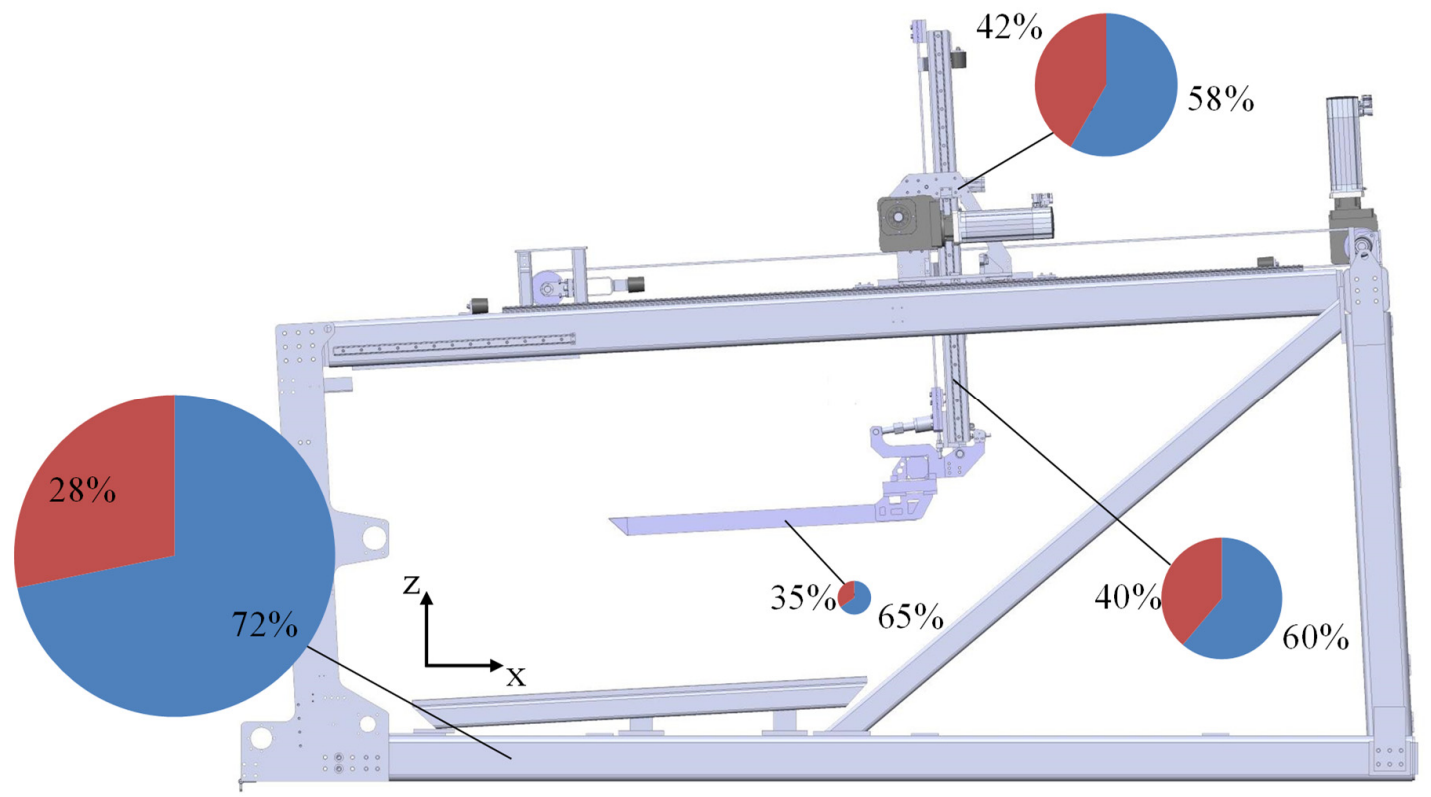

Figure 8. 2-axis processing machine with different mass-loss percentages (red area of circle) for the different assemblies (different circle diameters indicate different total assembly masses)

It can be seen that the percentages of the assembly mass to reduce also differ due to the different $\eta_{i}$ values of those assemblies. The descending order of optimization preference is thus directly derived from the energy-level factor, $\eta_{i}$, which is:

1. Horizontal cart $\left(\eta_{H C}=0.387\right)$

2. Vertical cart $\quad\left(\eta_{V C}=0.285\right)$

3. Operating fork $\left(\eta_{O F}=0.038\right)$

4. Frame $\quad\left(\eta_{F}=-0.290\right)$

The potential for optimization is dependent on the design of the assemblies, the material used, the costs and several other factors. However, the order of optimization presented here helps the designer to focus on the system parts which promise improved system characteristics if optimized with regard to lightweight.

\subsection{Discussion}

The results of the EDA facilitate an investigation of lightweight optimization potential at a superordinate level by showing the optimization ranking of the different assemblies or components respectively. On the level of the assembly itself, the EDA supports the designer by offering concrete mass-loss quantities. These quantities provide an orientation while actually reducing the mass through different strategies and methods must be done separately. While the presented method only takes state of motion of mass and mass distribution into account, lightweight optimization requires further considerations, for instance, the manufacturability and the costs of different components. A further development of the EDA must therefore consider a wider set of requirements to provide a more holistic lightweight support for the designer. Furthermore, another iterative loop of the EDA performed on the level of individual parts can provide more detailed information and increase the depth of the analysis. For instance, the frame can be examined as an individual part. One possible result may show that the upper horizontal metal beam has a higher optimization potential than the lower horizontal beam due to the higher potential energy. This may result in a material substitution for the upper beam alone in the form of a lighter material. 


\section{Conclusion and outlook}

This paper introduces a new method for lightweight design during the early phases of the product-design process called the Energy Distribution Analysis (EDA). The EDA enables the designer to analyze a product or a technical system in order to detect potential for lightweight optimization. Based on the information gained from the EDA, the lightweight optimization process can focus on the components that enable the product characteristics and performance to be improved if the mass is reduced. Consequently, the EDA closes the gap in the state of the art for lightweight methods with regard to the state of motion of mass, mass distribution and mass-dependent working principle.

The method was developed in science and theory. Although only applied to one real industrial product so far, the EDA still has to prove its revisability, comprehensibility, usefulness and flexibility in other projects. The feedback gathered will allow the method to be developed further.

One further development for a holistic lightweight design is to extend the method with regard to all axes so as to additionally analyze the balanced center of gravity (Figure 1). The next step is to integrate the Function Mass Analysis proposed by Posner et al. (2013) in order to cover an even wider area of lightweight design analytics with one single methodological approach.

\section{References}

Albers, A., Revfi, S. and Spadinger, M. (2017), "Extended Target Weighing Approach - Identification of Lightweight Design Potential for New Product Generations", Proceedings of the ICED 2017 / 21th International Conference on Engineering Design, Vancouver, USA, August 21 - 25, 2017, pp. 367 - 376.

Albers, A., Wagner, D., Ruckpaul, A., Hessenauer, B., Burkardt, N. and Matthiesen, S. (2013), "Target Weighing - A New Approach for Conceptual Lightweight Design in Early Phases of Complex Systems Development", Proceedings of the ICED 2013 / 19th International Conference on Engineering Design, Seoul, Korea, August 19 - 22, 2013, pp. 301- 310.

Feyerabend, F. (1990), Methodische Gewichtsreduzierung: Am Beispiel von Industrierobotern, PhD thesis, University of Paderborn, Paderborn.

Friedrich, H. E. (2013), Leichtbau in der Fahrzeugtechnik, Springer Vieweg Verlag, Wiesbaden.

Haldenwanger, H. G. (1997), Zum Einsatz alternativer Werkstoffe und Verfahren im konzeptionellen Leichtbau von PKW-Rohkarosserien, $\mathrm{PhD}$ Thesis, Technische Universität Dresden.

Henning, F. and Moeller, E. (2011), Handbuch Leichtbau, Carl Hanser Verlag, München.

Hering, E., Martin, R. and Stohrer, M. (2007), Physik für Ingenieure, Springer Verlag, Berlin, Heidelberg.

Keller, A. and Binz, H. (2009), "Requirements on Engineering Design Methodologies", Proceedings of the ICED 2009 / 17th International Conference on Engineering Design, Stanford, USA, August 24 - 27, 2009, pp. 317 329.

Klein, B. (2013), Leichtbau-Konstruktion, Springer Vieweg Verlag, Wiesbaden. https://doi.org/10.1007/978-3658-02272-3

Ponn, J., and Lindemann, U. (2011), Konzeptentwicklung und Gestaltung technischer Produkte, Springer Verlag, Berlin, Heidelberg. https://doi.org/10.1007/978-3-642-20580-4

Posner, B., Binz, H., and Roth, D. (2013), "Operationalisation of the Value Analysis for Design for Lightweight: The Function Mass Analysis”, Proceedings of the ICED 2013 / 19th International Conference on Engineering Design, Seoul, Korea, August 19 - 22, 2013, pp. 144 - 154.

Posner, B., Keller, A., Binz, H. and Roth, D. (2012), "Holistic Lightweight Design for Function and Mass: A Framework for the Function Mass Analysis", Proceedings of the DESIGN 2012 / 12th International Design Conference, Dubrovnik, Croatia, May 21 - 24, 2012, The Design Society, Glasgow, pp. 1071 - 1080.

Torenbeek, E. (1976), Synthesis of subsonic airplane design, Delft University Press, Delft. https://doi.org/10.1007/978-94-017-3202-4

Trzesniowski, M. (2014), Rennwagentechnik, Springer Vieweg Verlag, Wiesbaden. https://doi.org/10.1007/978$3-658-04919-5$

VDI (2010), 2800 Value Analysis, Beuth Verlag, Düsseldorf.

Felix Laufer, M.Sc.

University of Stuttgart, IKTD

Pfaffenwaldring 9, 70569 Stuttgart, Germany

Email: felix.laufer@iktd.uni-stuttgart.de 